\title{
ICRF Operation with Improved Antennas in ASDEX Upgrade with W wall
}

\author{
V Bobkov ${ }^{1}$, M Balden ${ }^{1}, \mathbf{R}$ Bilato $^{1}$, F Braun ${ }^{1}, \mathbf{R}$ Dux $^{1}$, \\ A Herrmann ${ }^{1}$, H Faugel $^{1}, \mathrm{H}$ Fünfgelder ${ }^{1}$, L Giannone $^{1}$, \\ A Kallenbach ${ }^{1}, \mathbf{H}$ Maier $^{1}, \mathbf{H}$ W Müller ${ }^{1}, \mathbf{R}$ Neu, \\ J-M Noterdaeme ${ }^{12}$, Th Pütterich ${ }^{1}$, V Rohde $^{1}$, N Tsujii ${ }^{1}$, \\ F Zeus ${ }^{1}, \mathrm{H}$ Zohm $^{1}$ and ASDEX Upgrade team \\ 1 Max-Planck-Institut für Plasmaphysik, EURATOM Association, Boltzmannstr. 2, \\ 85748 Garching, Germany \\ 2 Department of Applied Physics, Gent University, 9000 Gent, Belgium
}

\begin{abstract}
Experiments with boron-coated side limiters of two antennas operated together in 2012 showed that the side limiters are responsible to more than half of the increased $\mathrm{W}$ content in the plasma. Together with the contribution from the other limiter tiles, not replaced in 2012, the limiters accounts for at least $2 / 3 \mathrm{rds}$ of the $\mathrm{W}$ content. A modified test two-strap ICRF antenna in ASDEX Upgrade with broad limiters and narrow straps has shown an improved operation with full $\mathrm{W}$-wall in 2011/2012 campaigns with up to a $40 \%$ lower rise of $\mathrm{W}$ concentration allowing more stable operation at low deuterium gas injection rate. Limiter spectroscopy measurements indicate up to a $40 \%$ reduction of the rise of the $\mathrm{W}$ sputtering yield during ICRF power, measured under the assumption of negligible influence of geometry variations and reflections on the measurements. The boron limiters on two antennas together with the improved broad-limiter antenna allowed a successful ICRF operation in 2012. As a part of long-term strategy of antenna design development, two threestrap antennas with phase and power balance control for reduction of $E_{\|}$are planned for installation in the future.
\end{abstract}




\section{Introduction}

Operation of ICRF (Ion Cyclotron Range of Frequencies) antennas in magnetic fusion experiments is often accompanied by enhanced plasma-surface interactions. These become more problematic in high- $Z$ machines, such as the full tungsten (W) ASDEX Upgrade (AUG), where the $\mathrm{W}$ released from the wall during the ICRF operation contributes to radiation losses from the central plasma. These losses become substantial if the $\mathrm{W}$ concentration exceeds $5 \cdot 10^{-5}$, which is usually the case during ICRF operation in AUG with moderate or low deuterium gas injection rate. A significant part of the ICRF-specific plasma wall interactions in AUG is thought to be a result of RF enhanced sheaths due to the existence of $E_{\|}$, the parallel component of RF electrical field near the antenna [1]. This field contributes to elevated sheath potentials which can directly influence the $\mathrm{W}$ sputtering. It can affect as well as depend on the plasma convection in the scrape-off-layer [2]. The resulting effect of the near-field on the $\mathrm{W}$ release is difficult to characterize, because the exact conditions, such as small-scale geometry of magnetic field line connections, play a significant role $[1,2]$, whereas the diagnostic capabilities are limited.

In AUG, two strategies on establishing the compatibility of ICRF antennas with the $\mathrm{W}$ wall are being pursued.

The short-term strategy on making the ICRF operation compatible with the W wall at the low gas injection rate conditions in AUG, makes use of low-Z materials in the vicinity of ICRF antennas by the implementation of boron coatings on the antenna limiters. This does not solve the general problem of the ICRF-related plasma-wall interaction, but helps AUG to operate with ICRF in a wider range of conditions until the improvements of antenna design prove to be sufficient for the compatibility with $\mathrm{W}$ wall.

The long-term strategy is based on changes of ICRF antenna design. The connection of the $\mathrm{W}$ release to the $E_{\|}$field is taken as a working hypothesis for the antenna design process and the work presented in this paper. As a criterion for the design, an overall reduction of the $E_{\|}$field calculated with the help of finite-elements EM calculations was chosen.

Within the working hypothesis, the overall reduction of the $E_{\|}$field represents a relatively safe (though limited) approach to design an antenna, before an advanced criterion is developed by theoretical studies such as $[3,4]$ and benchmarked. This approach is used rather than a reduction of the integrated $E_{\|}$on a family of magnetic field lines, because of the known complexity of the antenna-plasma-wall interactions $[5,6]$. Recently, the popular criterion of reduction of the integrated $E_{\|}$ on the "long" field lines (the open field lines which pass in front of an antenna) has been questioned by the experimental observations in Tore Supra and in Alcator C-Mod.

In Tore Supra, modifications of the Faraday screen have been made [7] in order to affect the pattern of the parallel RF currents on the antenna frame and this way minimize the integrated $E_{\|}$on the long field lines. However, experimental data [8] 
suggests that the minimization of the integrated $E_{\|}$on the long field lines is not enough to reduce the wave-plasma interactions on the antenna itself. The data questions the criterion of minimization of the integrated $E_{\|}$on the long field lines.

In Alcator C-Mod for new field-aligned antenna [9], the guiding design principle was field line symmetry. This design results in minimization of the integrated $E_{\|}$due to cancellation along the long field lines and a modest reduction of local $E_{\|}$field. The new antenna has shown an improved operation by many criteria, including a significantly decreased impurity content during its operation. On the other side, the measurements of plasma potentials by gas-puff imaging [10] revealed qualitative inconsistencies between the potentials and the theoretically predicted values of the integrated $E_{\|}$on the long field lines [11].

In the AUG antenna design process, the criterion of the overall reduction of $E_{\|}$is realized by minimization of peak amplitude of $E_{\|}$averaged along the long field lines: $\left\langle E_{\| 0}\right\rangle=\frac{1}{L_{f}} \int E_{\| 0} d l$, where $E_{\| 0}$ is a local peak amplitude of $E_{\|}$and $L_{f}=\int d l$ is the length of the long field line within the boundaries of the calculation frame. The value of $\left\langle E_{\| 0}\right\rangle$ can be minimized by using the guidelines described in [1].

For antenna comparison in AUG presented in this paper, we rely mostly on the data of: (a) - W content in the plasma measured using the interpretation of spectral intensities of highly charged W ions [12]; (b) - the W sputtering yield at the antenna limiters measured by local limiter spectroscopy based on the interpretation of WI emission [13]. Diagnostics is being developed in AUG to make retarding field analyzer measurements [14]. Results on the antenna characterization from single location will be reported in [15]. In the future measurements with two probes at two locations simultaneously should allow a comparison of structures of plasma potential mapped to two different antennas.

At first, this paper describes the short-term strategy of using the low-Z materials in the antenna vicinity in section 2 . Then the long-term strategy is discussed in section 3 , where a detailed analysis of the experimental results reported briefly in [16] is presented together with the future plans for antenna developments in AUG.

\section{Short-term strategy: boron coated antenna limiters}

The standard $\mathrm{H}$ minority scheme with $(0, \pi)$ strap phasing was utilized for all the experiments reported in the paper. Four two-strap antennas (a1 to a4) in pairs are connected using $3 \mathrm{~dB}$ hybrids to the RF generators.

Previous studies [2] have shown that the antenna limiters play a dominant role as a W source during application of ICRF power. To increase the operational window for the ICRF system in AUG, the side limiters of $a 1$ and $a 2$ (see Fig. 1(a)) were coated by a $50 \mu \mathrm{m}$ thick layer of boron prior to the installation in the vessel. The coatings were produced by vacuum plasma spraying on fine grain graphite, similar as it was done in Alcator C-Mod for the molybdenum antenna limiters [17]. Boron is used in AUG during boronizations, therefore no new material was introduced into the machine. 
(a)

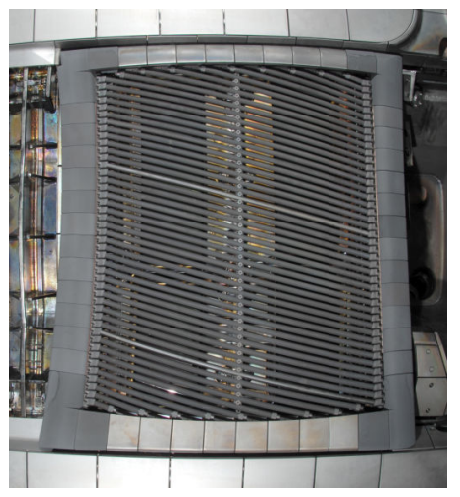

(b)

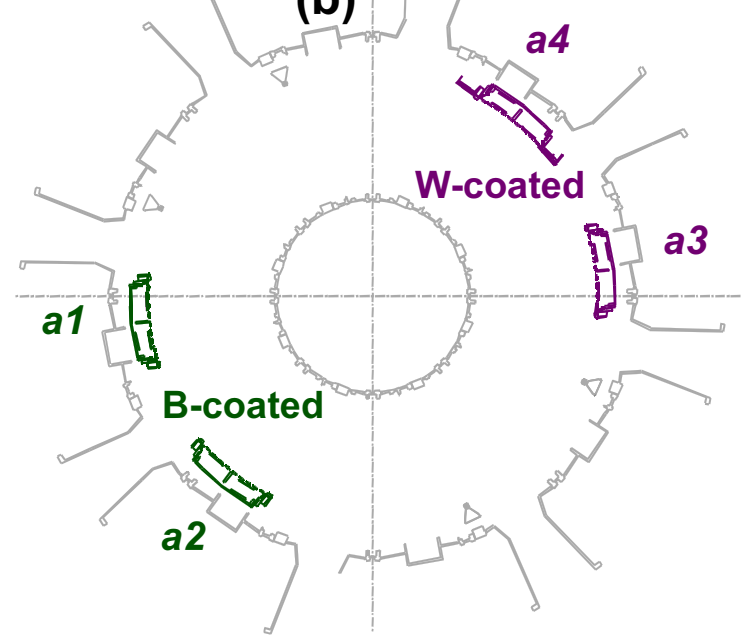

Figure 1. (a) Antenna a1 with the boron-coated side limiters. (b) Antenna arrangement with paired antennas a1 and a 2 equipped with boron-coated side limiters.

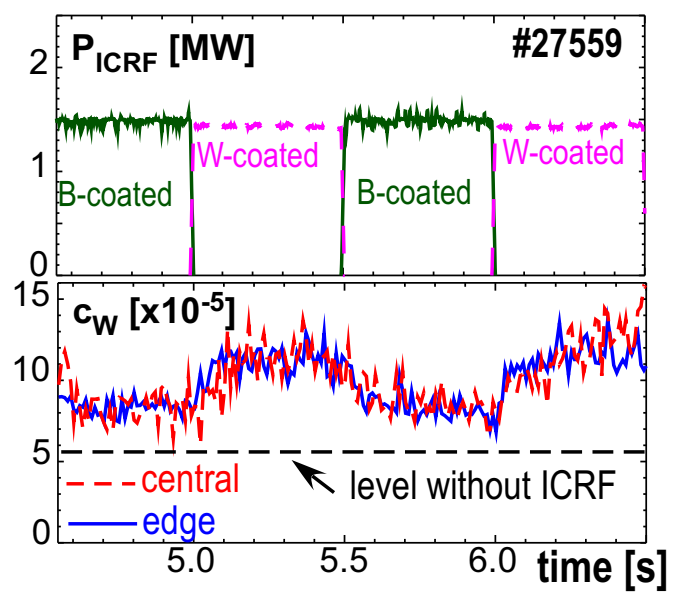

Figure 2. Comparison of operation of the antennas with boron-coated side limiters and those with full $W$-coated limiters.

For the most part of the 2012 experimental campaign, both antennas $a 1$ and $a 2$ with the boron-coated limiters were connected as a pair(see Fig. 1(b)) within the $3 \mathrm{~dB}$ hybrid connection scheme, whereas $a 3$ and $a 4$ were connected as the other pair. This allowed a discrete operation of the antennas with the boron-coated limiters and of the antennas with the $\mathrm{W}$-coated limiters. The standard $\mathrm{H}$ minority scheme with $(0, \pi)$ strap phasing was used.

The difference in production of $\mathrm{W}$ between the two antenna pairs was clearly visible in the experiment. Figure 2 shows the comparison of the two antenna pairs in terms of W concentration $c_{W}$ for the case of the scenario with $I_{p}=1 \mathrm{MA}, B_{t}=-2.5 \mathrm{~T}, P_{N B I}=7.5$ $\mathrm{MW}, P_{E C R H}=2.5 \mathrm{MW}, P_{I C R F}=1.5 \mathrm{MW}$ per antenna pair at the frequency of 36.5 
MHz. A very similar picture is observed with $B_{t}=-2.0 \mathrm{~T}$ at $30 \mathrm{MHz}$. The side $\mathrm{W}$ limiters account for more than a half of the increase of $\mathrm{W}$ concentration. Based on the local spectroscopic observations on the upper row of the limiters at $a 3$, the contribution of the upper and the lower rows of the limiters, not replaced by the boron-coated limiters at $a 1$ and $a 2$, can be roughly estimated to be $1 / 4$ to $1 / 3$ of that from the side limiters. The total effect of the antenna limiters on the $\mathrm{W}$ source is thus even stronger. Even more so, considering the fact that the broad-limiter antenna $a 4$, one of the two antennas with the $\mathrm{W}$ limiters, leads to lower increase in $c_{W}$ (see subsections 3.2 and 3.3 below). Therefore the observations confirm the dominant role of the antenna limiters in the $\mathrm{W}$ source associated with the ICRF power in AUG.

The boron coatings have shown good durability during the high-power AUG experiments. Surfaces of all limiter tiles, except the leading edges of tiles at the upper corners where the heat loads were the highest, showed almost no changes. The surface of the upper corner tiles was modified, forming crystals of boron in several locations. Nevertheless this did not jeopardize the machine operation nor the improved ICRF operation.

The improved ICRF operation of the antennas $a 1$ and $a 2$ with the boron-coated limiters allowed multiple ITER-relevant experiments using the ICRF power [26, 27, 28] during the 2012 experimental campaign. The modest reduction factor of the increase of W concentration of about two for the antennas has lead to significant extension of the operational window. This encourages the conservative long-term strategy of antenna development adapted in AUG which aims at similar factors of improvement.

\section{Progress on long term strategy of antenna design development}

The long-term strategy concentrates at building an antenna with minimized sputtering on W-coated antenna limiters. Under the working hypothesis of the $E_{\|}$field being responsible for the $\mathrm{W}$ sputtering, the design criterion of overall reduction of $E_{\|}$in terms of the averaged peak amplitudes along the field lines $\left\langle E_{\| 0}\right\rangle$ is applied. The field is calculated using the the finite-elements codes such as HFSS $\dagger$ and TOPICA [18]. The antenna design was mainly developed using the HFSS code with the plasma loading modeled by a lossy dielectric. Variations of antenna design were then examined and confirmed using the TOPICA code which uses a plasma model for the loading.

Figure 3 presents the development strategy of the antenna design by showing the results of the HFSS code calculations. The guidelines described in [1] were used which rely on a reduction of the $E_{\|}$field in front of the antenna mainly by minimizing the RF image currents at antenna limiters. This is done by several methods. Firstly, the outer antenna straps are bias-cut (see indications (1) in Fig. 3) to increase the distance between the straps and the antenna limiters and minimize the negative impact on the antenna loading due to narrower straps. Secondly, larger surfaces (low impedance conditions) for the RF image currents to flow and short-circuit themselves are introduced either behind

$\dagger$ HFSS (High Frequency Structure Simulator), http://www.ansys.com 


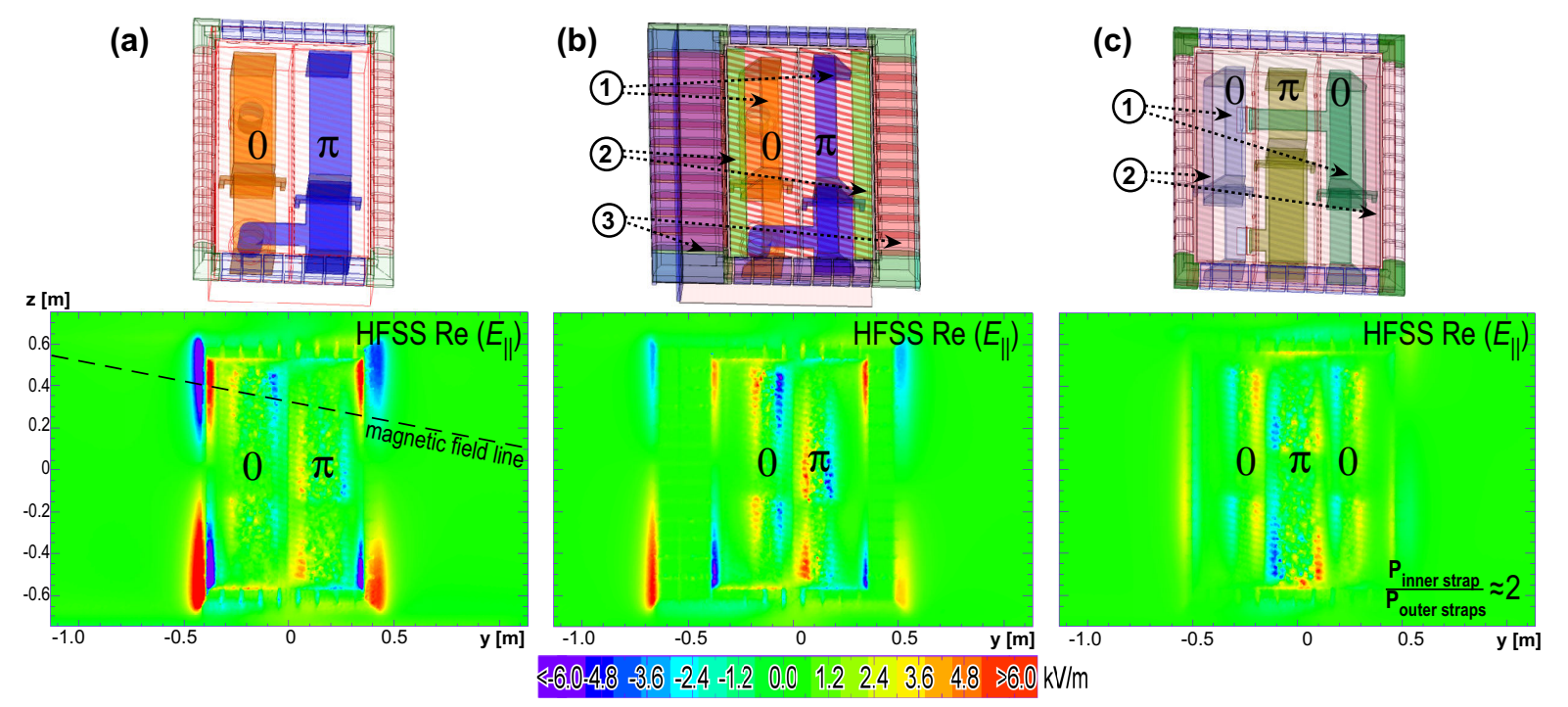

Figure 3. Calculations of $E_{\|}$by HFSS in front of antenna, normalized to the power of $1 \mathrm{MW}$ crossing perpendicular to antenna plane, all for dipole phasings. a) original AUG ICRF antenna; b) broad-limiter antenna; c) three-strap antenna. Indicated as (1): bias-cut straps; as (2): additional image current carrying surfaces; as (3): broad limiters.

the Faraday screen (indications (2) in Fig. 3) or by making antenna limiters broader (indication (3) in Fig. 3). Thirdly, the balance between the $(0, \pi)$-phased contributions of the image current at the limiters is optimized.

The HFSS field maps plotted in Fig. 3 show the $E_{\|}$fields normalized to $1 \mathrm{MW}$ of $\mathrm{RF}$ power crossing the antenna plane. The feeding scheme in the calculations was chosen such that the $E_{\|}$fields are predominantly real for the dipole phasing of interest.

The first, low-cost step of the strategy with relatively small antenna changes, was the modification of one two-strap antenna. This was done prior to the 2011 experimental campaign. The antenna is referred as a "broad-limiter" antenna (see Fig. 3(b) for corresponding HFSS model and Fig. 5 for the photo of the real antenna). The more aggressive antenna changes resulting in a design of a new three-strap antenna (see Fig. 3(c) and section 3.4) are planned as the next step in the future.

The reduction of $E_{\|}$can be judged from Fig. 4 where values of $\left\langle E_{\mid 00}\right\rangle$ are presented for the three antennas types. The broad-limiter antenna design reduces the $E_{\|}$field at the antenna limiters. At the same time, the $E_{\|}$component of the capacitive fields at the antenna straps increases on small areas in front of the straps. This happens at the high voltage regions of the straps, because of a worse antenna loading than for the original design. The total effect in terms of $\left\langle E_{|| 0}\right\rangle$ is positive. For the three-strap antenna, the highest fields are concentrated in front of the high voltage region of the middle strap. It remains to be shown whether these capacitive fields with a good symmetry along the magnetic field lines play a different role in impurity release at the limiters than the $E_{\|}$ field at the limiters. The latter is reduced on a large area in the calculations. 


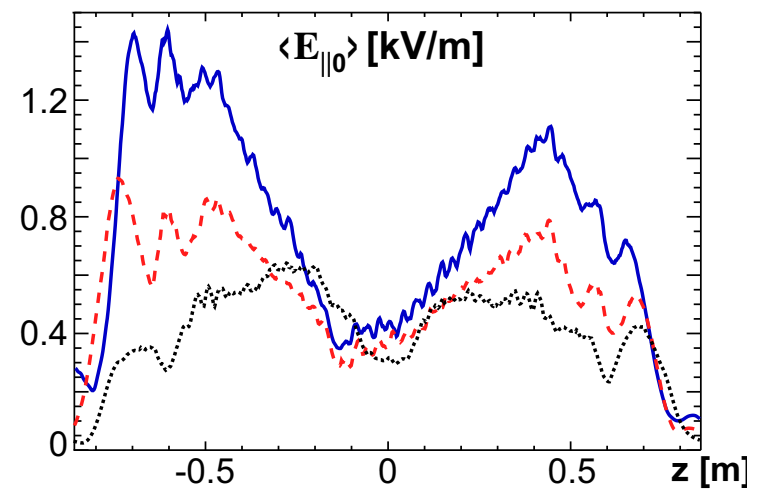

Figure 4. Calculated averaged peak amplitudes of $E_{\|}$along the field lines $\left(\left\langle E_{|| 0}\right\rangle\right)$ along the vertical axis at the middle of antenna $(y=0)$. Solid blue curve - original design, dashed red curve - broad limiter antenna, dotted black curve - three strap antenna.

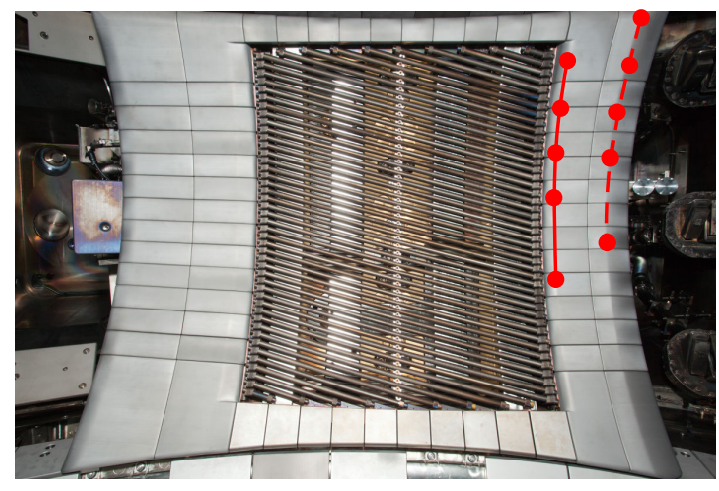

Figure 5. The broad-limiter antenna in AUG with spots of spectroscopic observation.

It should be noted that the broad antenna limiters increase the area of the plasmafacing components coated by $\mathrm{W}$ in the very vicinity of the antenna. This is the drawback of such antenna design. It is to some extent compensated by the fact that the impinging particle flux is distributed over a larger surface of the broad limiters. Under these conditions, the limiter surfaces closer to the antenna straps confront lower particle fluxes than the surfaces further away from the straps (see subsection 3.3).

Within this paper, the field maps are treated as guidelines for the antenna development. As described in [1], a direct comparison of the maps with the W sputtering patterns would require strong assumptions. A step towards better comparison of antennas can be done by measuring plasma potentials via probe techniques [14] which are under development in AUG [15]. Once such measurements become available for two antennas simultaneously, they will be reported elsewhere.

Antennas $a 1$ and $a 3$ ( $a 2$ and a4) located opposite to each other (see Fig. 6) formed antenna pair(s) throughout the 2011 campaign to allow independent feeding of neighboring antennas. As only antenna $a 4$ has been modified, for some the experiments, which are described in section $3.2, a 1$ and $a 2$ were mismatched to allow direct comparison 


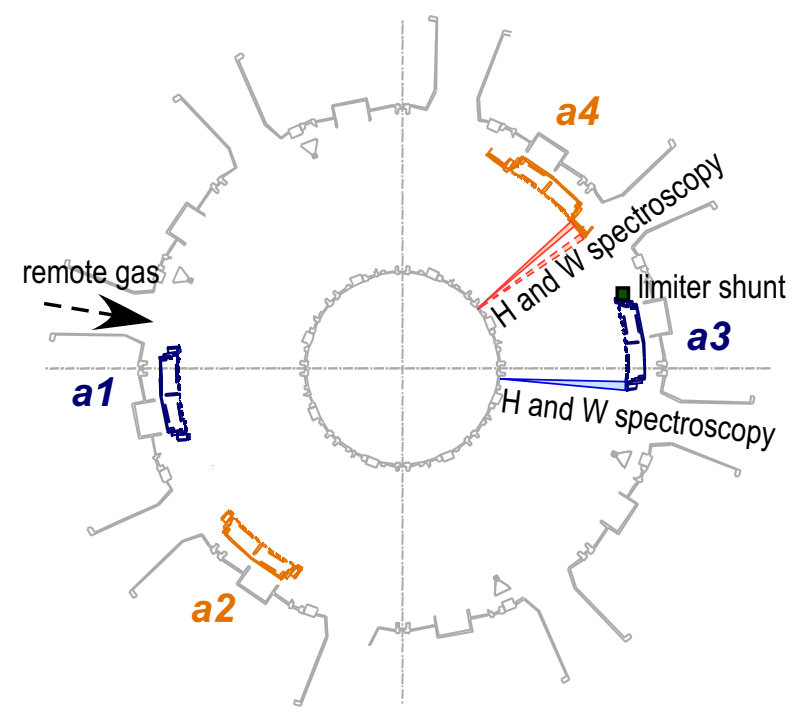

Figure 6. Toroidal arrangement of antennas and observations in AUG in 2011.

between $a 3$ (original) and $a 4$ (broad-limiter). However, this limited the available ICRF power to only about $500 \mathrm{~kW}$ per antenna, because of the issues with reflected power at the RF generators, due to the low efficiency of the $3 \mathrm{~dB}$ hybrid insulation scheme at strongly misbalanced reflections from the antennas.

\subsection{Equality of conditions for operation of original and broad-limiter antennas}

To ensure the equality of conditions for operation of the original and the broad-limiter, a number of parameters need to be controlled.

Antennas $a 3$ (original design) and $a 4$ (broad-limiter design) are the antennas used for comparison throughout this section of the paper. Radial coordinates of several poloidal locations of one poloidal side limiter per antenna have been measured before the experimental campaign. These were found to be equal for both $a 3$ and $a 4$ antennas within the accuracy of the measurements of $2 \mathrm{~mm}$. The configuration of antenna and guard limiters in AUG provides a similar profile of the connection lengths outside the original and the broad-limiter antennas.

With central temperature and density profiles fixed, the location of the gas injection plays a significant role for the $\mathrm{W}$ sputtering. This is shown in Fig. 7 for the experiment conducted in 2009 [19], for which the same antenna pairing was used as in Fig. 6, albeit $a 4$ was still of original design. Values of the $\mathrm{W}$ influx $\Gamma_{W}$ and the effective $\mathrm{W}$ sputtering yield $Y_{W}$ were measured spectroscopically at the poloidal side limiter of $a 4$. For the ICRF-only discharges of interest $\Gamma_{W} \approx \Delta \Gamma_{W}$ and $Y_{W} \approx \Delta Y_{W}$, where $\Delta \Gamma_{W}$ and $\Delta Y_{W}$ are increments due to ICRF. Both $\Gamma_{W}$ and $Y_{W}$ are reduced, especially at vertical position $z>0.1$, when $D_{2}$ working gas injection local to the antenna is used compared to the gas injected with the same rate remotely (see [19] for details of gas injection). Thus, the $D_{2}$ gas injection remote with respect both to $a 3$ and $a 4$ was chosen for the antenna 


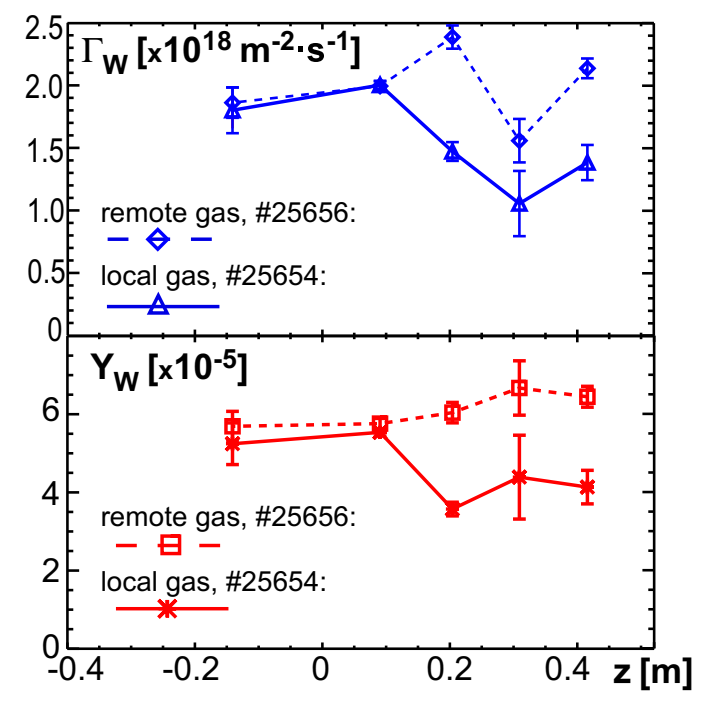

Figure 7. Influence of gas injection location on the $W$ sputtering pattern on antenna limiters ( $\Gamma_{W}$ on upper graph and $Y_{W}$ on lower graph, standard errors).

comparison.

To minimize influences of boron layers on the limiters (in particular their inhomogeneities) which are deposited during boronizations in AUG on the characterization of $\mathrm{W}$ release during ICRF, experiments were conducted at least 100 plasma shots after the boronizations.

\subsection{Comparison of original and broad-limiter antenna in operation}

To test the antennas, an H-mode scenario with $P_{N B I}=5 \mathrm{MW}$ at a magnetic field $B_{t}=-2.0 \mathrm{~T}$ and plasma current $I_{p}=0.8 \mathrm{MA}$ with constantly decreasing $D_{2}$ gas injection rate $\Gamma_{D 2}$ was used with ICRF power at $30 \mathrm{MHz}$ for central heating. In $\mathrm{H}$ modes in the full-W AUG under such conditions, a lower threshold of $\Gamma_{D 2}$ exists, below which the $\mathrm{W}$ accumulation in the plasma develops. The point of the accumulation can be easily determined by observing the central and the edge lines of sight of the bolometers (see lower two graphs of Fig. 8). In Fig. 8, vertical dashed lines show the $\mathrm{W}$ accumulation thresholds for the shot where only the broad-limiter antenna was used (red) and for the shot where only one original antenna was powered (blue). The broadlimiter antenna allows operation at lower $\Gamma_{D 2}$ without the $\mathrm{W}$ accumulation, with other parameters fixed.

Interestingly, a comparison with pure NBI heated discharges in the same scenario shows that the application of ICRF power using the broad-limiter antenna prolongs the phase without $\mathrm{W}$ accumulation to lower $\Gamma_{D 2}$ for this scenario, as is expected from the application of central RF heating without the detrimental effect of the $\mathrm{W}$ source. At the same conditions, the ICRF power from the original antenna a3 does not affect the duration of the $\mathrm{W}$-accumulation-free phase, exposing the negative influence of the $\mathrm{W}$ 


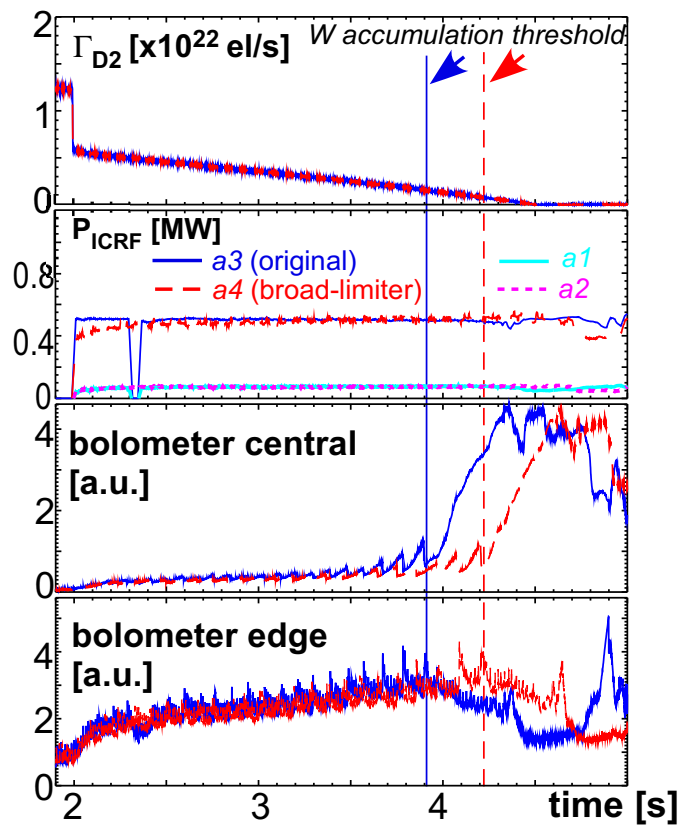

Figure 8. Comparison of antennas by the threshold of $\Gamma_{D_{2}}$ for $W$ accumulation (vertical lines) for \#26745 (dashed, red, a4) and \#26746 (solid, blue, a3).

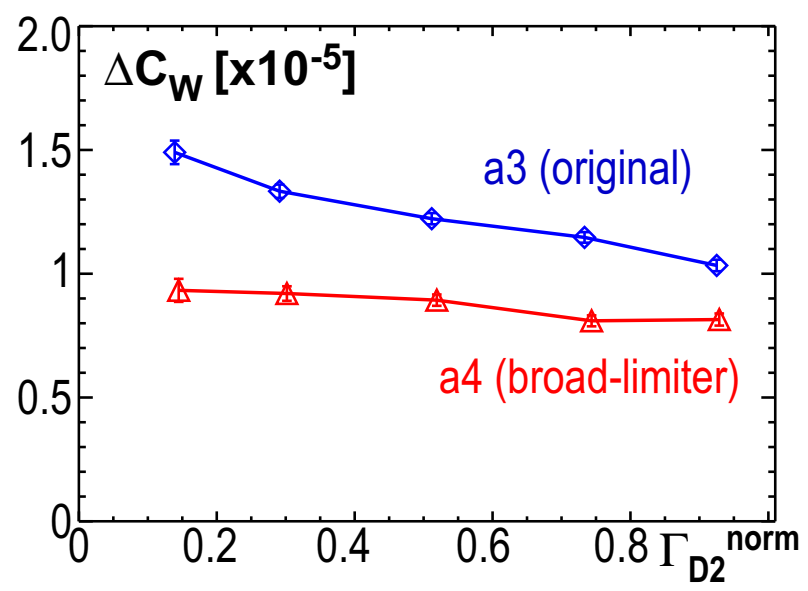

Figure 9. $\Delta c_{W}$ due to $0.5 \mathrm{MW}$ of ICRF power from original (blue diamonds) and the broad-limiter antennas (red triangles).

source associated with ICRF at these conditions of low $\Gamma_{D 2}$.

The antenna comparison was successfully reproduced several times during the 2011 experimental campaign. The data from this series of discharges are summarized in Fig. 9, where the change of $\mathrm{W}$ concentration $\Delta c_{W}$ due to ICRF is shown with respect to $\Gamma_{D 2}$. The latter is normalized to allow the use of the data taken at different machine conditions during the 2011 campaign: $\Gamma_{D 2}^{n o r m}=\left(\Gamma_{D 2}-\Gamma_{D 2}^{W a c c u m}\right) /\left(\Gamma_{D 2}^{\text {start }}-\Gamma_{D 2}^{W a c c u m}\right)$, where $\Gamma_{D 2}^{\text {start }}$ is a maximum value of $\Gamma_{D 2}$ at the beginning of ramp-down of the gas injection rate and $\Gamma_{D 2}^{W a c c u m}$ is the $\mathrm{W}$ accumulation threshold of $\Gamma_{D 2}$ in the discharges with the original 


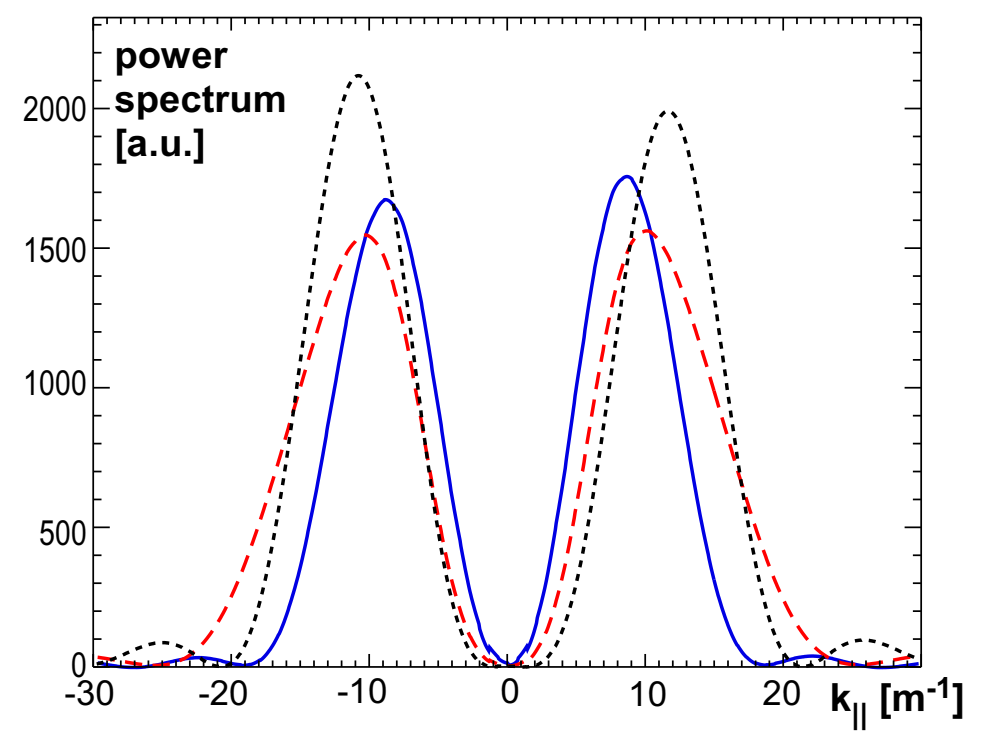

Figure 10. $k_{\|}$power spectra at antenna midplane based on toroidal $R F$ magnetic field from the HFSS antenna calculations: solid blue curve - original design, dashed red curve - broad limiter antenna, dotted black curve - three strap antenna.

antenna. Thus, value of $\Gamma_{D 2}^{n o r m}=0$ corresponds to the $\mathrm{W}$ accumulation threshold of the original antenna, whereas $\Gamma_{D 2}^{n o r m}=1$ corresponds to the maximum value of $\Gamma_{D 2}$. The data plotted is limited to $\Gamma_{D 2}^{n o r m}>0.1$ to show stationary conditions only. It can be seen that $\Delta c_{W}$ is up to $40 \%$ lower for the broad-limiter antenna than for the original antenna at a fixed gas injection rate. At the low gas injection rates $\Delta c_{W}$ for $a 4$ is lowest with respect to that for the original antenna.

Thus, despite the increased area of the antenna plasma facing components coated by $\mathrm{W}$, and even at relatively low $\mathrm{RF}$ power of $\approx 500 \mathrm{~kW}$ used per antenna, the modified antenna behaves consistently better in terms of the balance between the central heating and the $\mathrm{W}$ released during its operation.

One could argue that the improved performance of the broad-limiter antenna is due to an increased wave heating efficiency in the plasma core in the case of hydrogenminority scenario. In Fig. 10 the $k_{\|}$spectra of the antenna designs from Fig. 3 are presented. These are calculated based on the HFSS data for the cases of different antennas with the same lossy dielectric loading. The figure shows that the $k_{\| \text {-spectrum }}$ peaks at slightly higher $k_{\|}$of $9.5 \mathrm{~m}^{-1}$ for the broad-limiter antenna (red dashed curve) compared to the corresponding peak at $k_{\|}=8.5 \mathrm{~m}^{-1}$ for the original antenna (dotted blue curve). However full-wave calculations with the TORIC code [20] show very similar power deposition profiles for the cases of the original and the broad-limiter antennas. Furthermore, 1D AORSA code [21] calculations result in the same lower single pass absorption for both antennas within the error bars of calculations. Taking into account the fact that the experimental data does not indicate variations of heating efficiency between the antennas, it is unlikely that the better behaviour of the broad-limiter 
antenna can be explained by the wave heating efficiency in the plasma core. On the other hand, effects of the variations of the $k_{\|}$spectrum (such as reduction of power at $k_{\|} \approx 0$ ) on the antenna interactions with the plasma edge cannot be excluded. These effects are not modeled by the codes mentioned.

Further differences in the antenna behavior can be seen using the local spectroscopic measurements of the $\mathrm{W}$ sputtering patterns at $a 3$ and $a 4$.

\subsection{Local spectroscopic measurements at original and broad-limiter antenna}

Prior to the installation of the broad-limiter antenna, the limiter spectroscopy measurements [2] resulted in practically equal values of $\Gamma_{W}$ and $Y_{W}$ for $a 3$ and $a 4$. The measurements were performed under the conditions very similar to those of the experiments described below.

For 2011, the lines of sight for the limiter spectroscopy at the broad-limiter antenna were chosen to match approximately the same poloidal locations as in [2]. However the observation spots cover the different profiles of the broad and the original limiters and thus are not equivalent any longer. The different limiter shapes result in significantly higher line intensities of all spectral lines which are measured at the outer row of measurements of the broad-limiter (see the spots on the dashed lines of sight in Fig. 5 and a "dashed" line in Fig. 6), compared to the inner row of $a 4$ and the row at $a 3$. This is observed both during ICRF and without ICRF and implies higher sensitivity of the measurements and/or higher absolute values of particle flux $\Gamma_{D}$ of impinging deuterons on the outer row of a4. A combination of factors can play a role: a) the "shadowing" effect by the broad limiters is present due to the longer connection lengths of magnetic field lines at the outer row of $a 4$ than at the inner row of $a 4$ and due to the upstream parallel plasma flows; b) the limiter shape yields smaller averaged clearance between the plasma and the surface of the $a 4$ broad limiter covered by an observation spot than at the row at $a 3$. To be less prone to the variations in the diagnostics geometry during the antenna comparison, we limit ourselves to considering $Y_{W}$, in particular its change $\Delta Y_{W}$ due to ICRF. In fact, $\Delta Y_{W}$ represents the most relevant quantity from the spectroscopic measurements to infer about the elevated sheath voltages due to ICRF [13].

In addition, the assumption needs to be made that the reflections from other plasma facing components which can influence intensities of some spectral lines do not influence the distribution of $\Delta Y_{W}$.

Figure 11 shows comparison of $\Delta Y_{W}$ values at $a 3$ (original) and a4 (broad-limiter) for the discharges with variation of $\Gamma_{D 2}^{n o r m}$ as in Fig. 9, when either $a 3$ or $a 4$ is operated correspondingly. The $\Delta Y_{W}$ values are averages over the rows of observations shown in Fig. 5. The broad-limiter antenna a4 shows lower $\Delta Y_{W}$, both for the inside and the outside rows of the lines of sight. These observations are in line with the observed differences in $\Delta c_{W}$ in Fig. 9.

To improve quality of the data set for $\Delta Y_{W}$, discharges with higher gas injection rates are used which correspond to $\Gamma_{D 2}^{n o r m}$ significantly higher than 1 . In this operational 


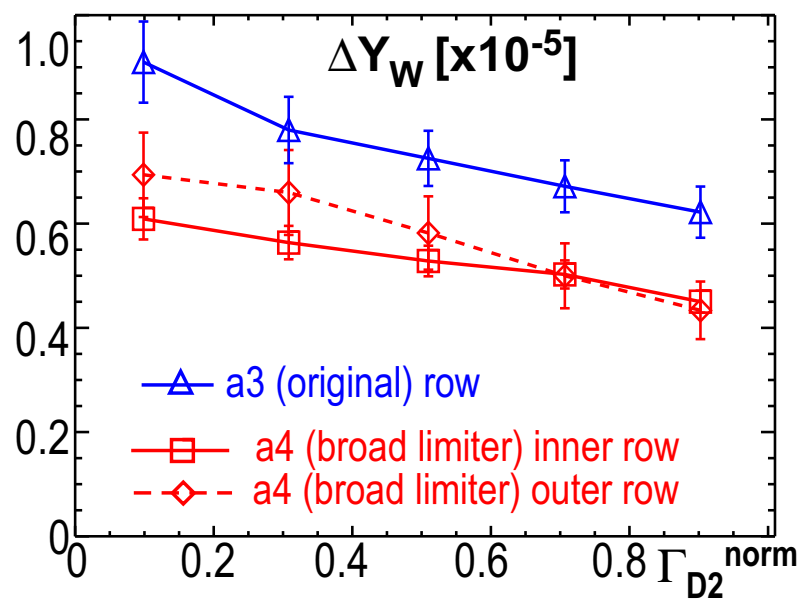

Figure 11. Comparison of $\Delta Y_{W}$ for a3 and a4 (inner and outer rows) depending on $\Gamma_{D 2}^{\text {norm }}$ (as in Fig. 9)

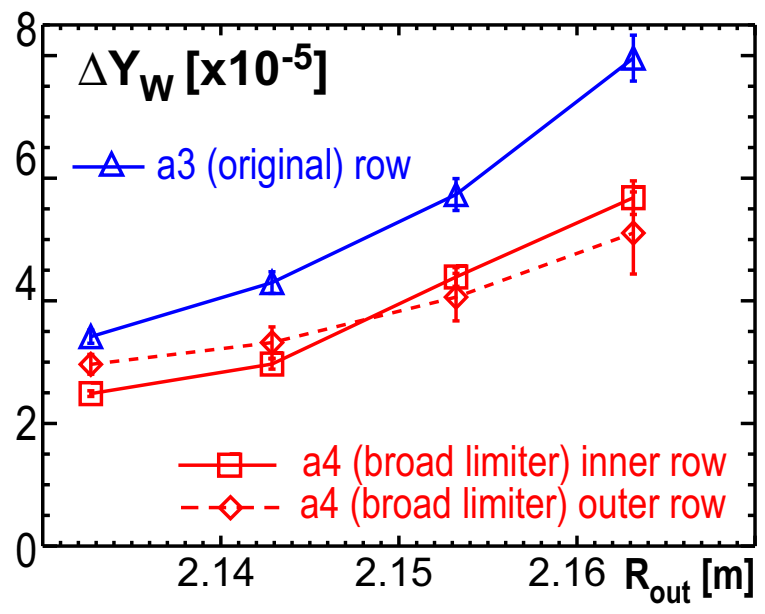

Figure 12. Comparison of $\Delta Y_{W}$ at active a3 and a4 depending on the plasma outermost position $R_{\text {out }}$ using data from \#\#26541-26544.

corner change of $c_{W}$ for the applied $P_{I C R F}=1 \mathrm{MW}$ is small, but the local effect of ICRF operation on $\Delta Y_{W}$ is well distinguishable. Power from all antennas, including $a 1$ and $a 2$ which do not interfere with local $\Delta Y_{W}$ measurements at $a 3$ and $a 4$ correspondingly, was used for discharges \#\#26541-26544 from the data set. The ICRF power was toggled between the antenna pairs $a 1 / a 3$ and $a 2 / a 4$. Figure 12 represents comparison of the $\Delta Y_{W}$ values for $a 3$ and $a 4$ (when those are active) vs. the plasma outermost position $R_{\text {out }}$. The broad-limiter antenna $a 4$ is characterized by up to $40 \%$ lower $\Delta Y_{W}$, both for the inside and the outside rows of the lines of sight. With smaller error bars and higher density near antenna compared to Fig. 11, values of $\Delta Y_{W}$ on the outer row are closer to those at the inner row in Fig. 12. At largest $R_{\text {out }}$, when the plasma is closest to the antenna, $\Delta Y_{W}$ measured at $a 4$ is lowest with respect to that at $a 3$. 


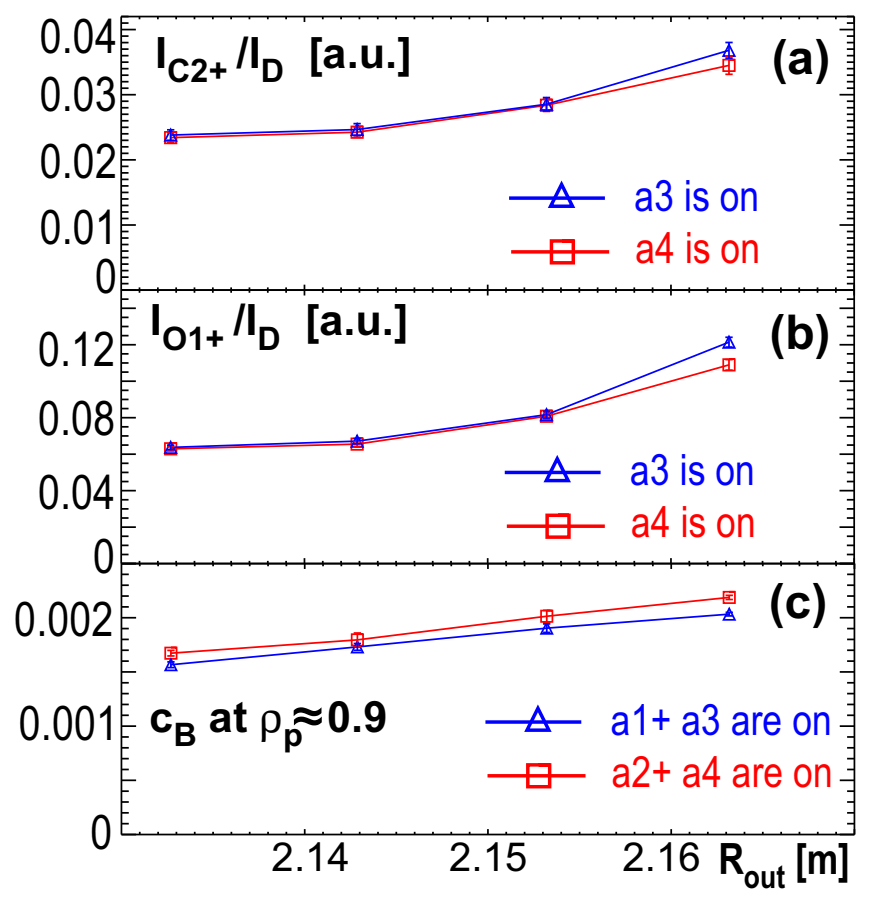

Figure 13. Comparison of light impurity content during operation of different antennas: (a) and (b) - ratios of $C^{2+}$ and $O^{1+}$ line intensities to $D$ line intensity measured by limiter spectroscopy and over all lines of sight at a 3 and a4; (c) - boron concentration in the confined plasma at $\rho_{p} \approx 0.9$ measured by CXRS. The data set is the same as in Fig. 12.

Another candidate to explain the antenna differences is a varying light impurity content. Light impurities dominate the W sputtering during ICRF at AUG [22], taking into account the typical ICRF-related plasma potentials in the range between 20 and $200 \mathrm{~V}$ observed in the far scrape-off layer of AUG $[1,15]$. The limiter spectroscopy has sufficient spectral resolution to measure intensities of $\mathrm{C}^{2+}$ at $406.9 \mathrm{~nm}$ and $\mathrm{O}^{1+}$ at $407.5 \mathrm{~nm}$ on the same set of lines of sight and simultaneously with the line intensities of neutral W (400.9 nm) and D (410.1 nm) used for the measurements of $\mathrm{W}$ and D influx and effective sputtering yield described above. Carbon and oxygen are among the main contributors to the $\mathrm{W}$ sputtering [1]. The ratios of the $\mathrm{C}$ and the $\mathrm{O}$ spectral line intensities to the $\mathrm{D}$ line intensity are indicative of local concentrations of $\mathrm{C}$ and $\mathrm{O}$ at the antenna limiters. Fig. 13(a)(b) shows the ratios of the line intensities for the data set presented previously in Fig. 12. The data is averaged over the whole set of lines of sights and the phases are distinguished when either antenna $a 3$ or $a 4$ is active. With the exception of the case at largest $R_{\text {out }}$, the line intensity ratios are the same. Therefore the tendency of the larger relative difference of $\Delta Y_{W}$ between $a 4$ and $a 3$ in the case of the largest $R_{\text {out }}$ from Fig. 12 could be interpreted as the influence of variation of $\mathrm{C}$ and $\mathrm{O}$ content on the $\mathrm{W}$ sputtering. However in general, variations in $\mathrm{O}$ and $\mathrm{C}$ content near the limiters cannot be made responsible for the differences in $\Delta Y_{W}$. This rests under the assumption that the data on the $\mathrm{C}^{2+}$ and $\mathrm{O}^{1+}$ line intensities is representative of 
the content of all ionization stages of $\mathrm{C}$ and $\mathrm{O}$ near the limiters. Variations of the same order of magnitude would be needed for concentrations $\mathrm{C}$ and $\mathrm{O}$ to explain the differences in $\Delta Y_{W}$ in Fig. 12 in the range of sheath potentials noted above.

Boron is another light impurity which participates in the $W$ sputtering [1]. An idea about the boron content can be obtained using the charge exchange recombination spectroscopy [23] (CXRS) which measures boron concentration in the confined plasma. Fig. 13(c) shows that the operation of the $a 2 / a 4$ antenna pair is characterized by marginally higher boron concentration. However this observation can be interpreted in two opposite ways. On one hand, the higher boron content results in a stronger W sputtering during a4 operation. This counteracts the observed improvements of the $a 4$ operation, indicating that the improvement could be stronger if the boron concentration was the same. On the other hand, the higher boron content might indicate a larger coverage by boron of the recessed surfaces at, near or on magnetic field line connections to $a 4$. This could be one of the reasons for the observed a4 improvement discussed in section 3.2, but is unlikely to explain the decrease of $\Delta Y_{W}$ at the antenna limiters. The antenna limiters are exposed to the high particle fluxes and their surfaces can be considered boron-free after more than 100 discharges after boronizations.

With the variations of the light impurity content being unlikely to explain the decrease of $\Delta Y_{W}$, the latter can be considered compatible with a decrease of $E_{\|}$. However the assumptions on the negligible influence of geometry variations and reflections on $\Delta Y_{W}$ as well the limited capabilities of other currently existing diagnostics in AUG, do not allow to confirm that the reduction of $\Delta Y_{W}$ is caused by the reduced $E_{\|}$. A number of other effects such as the influence of the limiter geometry on the plasma properties near antenna and the influence of $k_{\|}$on antenna-plasma interactions cannot be excluded as the reasons behind the differences between the antennas.

\subsection{Three-strap antenna design and plans}

Two completely new three-strap antennas are planned for installation in AUG in the near future. Figure 3(c) shows the calculations of the near-fields for the antenna which shows a reduction of $E_{\|}$. Figure 14 presents a CAD model of the three-strap antenna. The principle of the design is based on finding a minimization of image currents by balancing between the $(0, \pi)$-phased image current contributions, as has been shown for a four-strap antenna in [1]. For the three-strap antenna, the minimization of the image currents is done by controlling the phase and the power distribution between the outer straps and the inner strap. The issues connected to the different shapes of the side limiters will be eliminated, because the same shape of the side limiters as that for the original antenna will be used for the three-strap antennas. To monitor the balance of the RF currents, an array of RF and DC antenna shunts [2] will be mounted on the antennas limiters.

From Fig. 10 it can be seen, that $k_{\|}$spectrum for the three-strap antenna has the dominant $k_{\|}$values close to that of the broad-limiter antenna. As noted above, this 

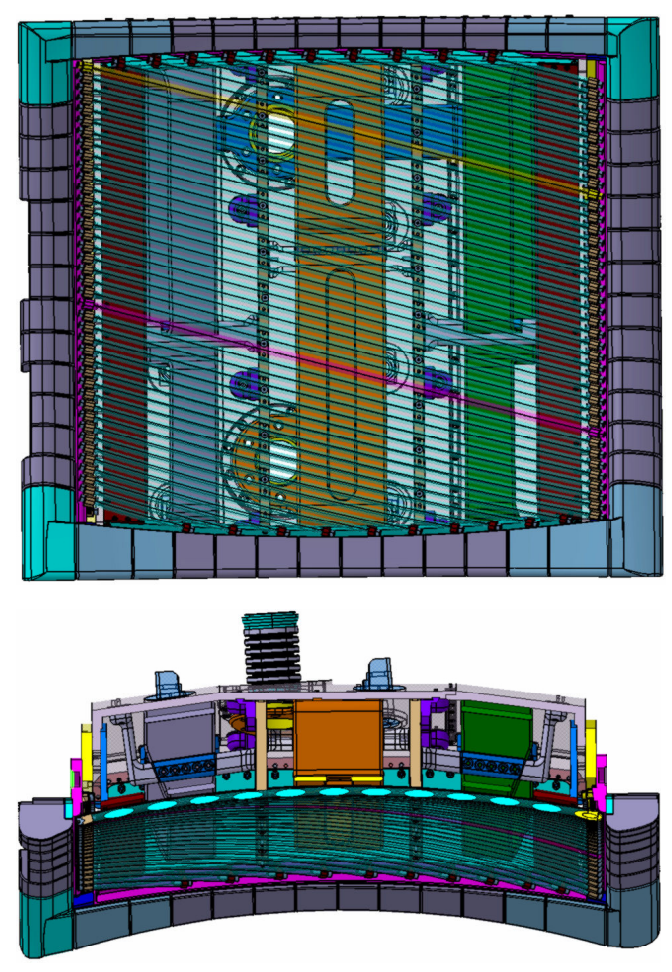

Figure 14. Front and top views of the three-strap antenna from CAD.

should result in similar absorption of the power in the plasma center. On the other hand, the lower values of $k_{\|}$close to 0 can aid reducing plasma-antenna interactions and are co-directional with improvements of compatibility with the $\mathrm{W}$ wall.

The installation of two antennas will allow the use of the full $3 \mathrm{~dB}$ insulation scheme without jeopardizing ICRF power and ELM tolerance. The upgrade includes also additional transmission lines and phase control hardware. A prototype of the new digital-based phase control system, described in [24], has been tested during experiments in 2011, by making a variation of phase between two neighboring antennas $a 3$ and $a 4$ during a plasma discharge. The results are presented in Fig. 15, where ICRF power, phase difference $\Delta \Phi$ between $a 3$ and $a 4, \Gamma_{W}$ at the outer line of sight at $z=0.2 \mathrm{~m}$ at $a 4$ as well as currents of the limiter shunt at $z=-0.1 \mathrm{~m}$ at $a 3$ (see Fig. 6 for overview of diagnostics) are shown. The value of $\Gamma_{W}$ on the single line of sight reacts sensitively on the changes of $\Delta \Phi$. A more detailed analysis of $\Gamma_{W}$ and $c_{W}$ [25] shows that the integral behavior of the $\mathrm{W}$ sources at $a 3$ and $a 4$ is less sensitive to the phase between the antennas, because the reaction is location dependent.

This location dependency is picked up by the limiter shunt currents at a3 presented at the lower graph of Fig. 15. Such measurements, in particular the DC current, show usually a correlation with locally measured $\Gamma_{W}$. For the case of Fig. 15 the shunt measurements occur not locally, but on approximately the same flux tube connecting the spot of spectroscopic observation at $a 4$ to the limiter at $a 3$. The shunt currents show anti-correlation to the values of $\Gamma_{W}$ at $a 4$, again indicating a location dependence of the 

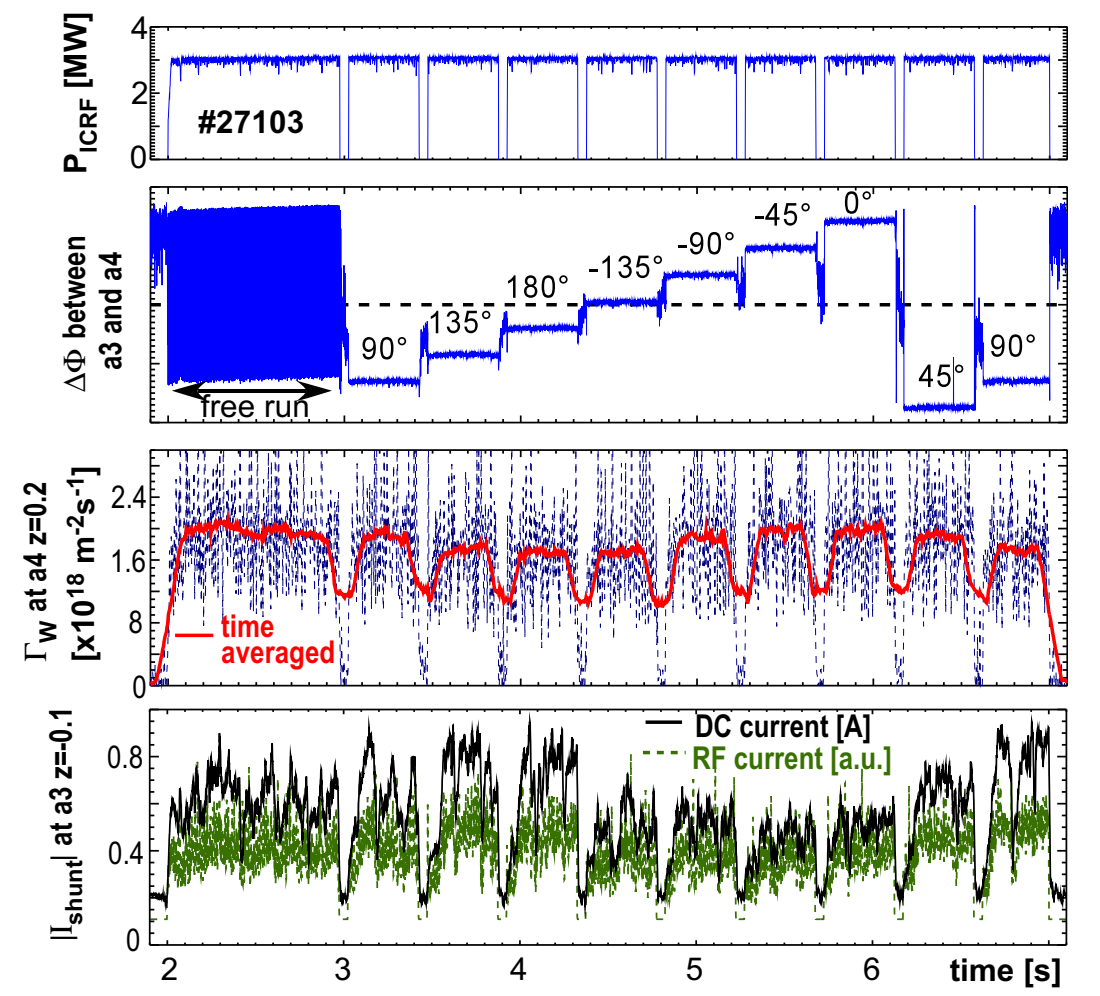

Figure 15. In-shot phase control between a3 and $a_{4}$, effect on $W$ source at single location at a4 and shunt currents at a 3 limiter.

effect of the phasing between the two antennas. As described in [25], measurements of $\Gamma_{W}$ at $a 3$ show almost no correlation to the change of phase, because those are located at the limiter further away from $a 4$.

A more sensitive behavior is expected for the new three-strap antennas during variation of phase between the straps. The phase will need to be actively controlled, ultimately together with the power distribution between the straps in real time, to minimize the total W source. Because of the expected strong load dependence on the changes of the strap phasing, the load tolerance of the $3 \mathrm{~dB}$ hybrid system will be crucial for the operation.

\section{Conclusions and outlook}

The boron-coated ICRF antenna side limiters have been tested in 2012. The experiments with the boron-coated limiters confirmed the dominant role of the limiters as $\mathrm{W}$ sources as well as provided a good possibility to conduct multiple ITER-relevant experiments with ICRF power in ASDEX Upgrade with $\mathrm{W}$ wall. It appears that already the reduction by a factor of two of the rise of $\mathrm{W}$ content during ICRF provides a significant extension of operational window for ICRF in ASDEX Upgrade. Development of ICRF design at ASDEX Upgrade aims at similar improvement factors.

The design development relies on the conservative approach of overall reduction of 
parallel component of RF electric field $E_{\|}$near antenna, under the working hypothesis that $E_{\|}$plays a dominant role in the impurity release during ICRF. A modified antenna design with broad-limiters and narrow straps has been tested in 2011. The experiments show improvements in compatibility with the full $\mathrm{W}$-wall for the antenna compared to the antennas of the original design. A more stable operation at low deuterium rate has been observed, as well as a lower change of $\mathrm{W}$ concentration in the plasma. Variations of the core wave heating efficiency due to modified $k_{\|}$spectrum are expected to be small and cannot explain the observations. The broad-limiter antenna is characterized by a lower change of sputtering yield at the limiters, measured under the assumption of negligible influence of variations in geometry and reflections. Data suggests that light impurity content cannot explain the differences of the $\mathrm{W}$ sputtering yield observed in the experiments. However the data obtained so far cannot confirm that the improvements are due to the decreased $E_{\|}$field near antennas, although no contradiction to this has been observed. The effects of the limiter geometry and $k_{\|}$on antenna-plasma interactions and on the antenna differences cannot be excluded. Development of probe diagnostics at ASDEX Upgrade which would allow to measure DC sheath potentials for two antennas simultaneously would shed more light on this topic in the future.

A completely new antenna design with two antennas three straps each is planned for the future with the aim to minimize the antenna near-fields to improve the $\mathrm{W}$ compatibility significantly. Besides the new design, the new antennas will have more experimental flexibility which will allow to improve understanding of the processes leading to impurity release during ICRF.

\section{Acknowledgement}

We would like to thank Dr. R. Fischer and Dr. R. McDermott for their help with the evaluation of plasma and impurity density profiles and Dr. L. Colas for discussions about the antenna codes.

\section{References}

[1] Bobkov V. et al., Nucl. Fusion 50 (2010) 035004.

[2] Bobkov V. et al., J. Nucl. Mater. 415, 1 (2011) S1005-S1008.

[3] D'Ippolito D.A. et al., AIP Conference Proceedings 1406 (19th RF Topical Conference on RF Power in Plasmas, 2011) 195.

[4] Colas L. et al., Phys. Plasmas 19, 092505 (2012).

[5] Myra J.R. et al., Nucl. Fusion 46 (2006) S455.

[6] D'Ippolito D.A. and Myra J.R., J. Nucl. Mater. 415, 1 (2011) S1001-S1004.

[7] Mendes A. et al., Nucl. Fusion 50 (2010) 025021.

[8] Colas L., J. Nucl. Mater., 438, 1 (2013) S330-S333.

[9] Wukitch S. et al., 2012 Proc. 24th Int. Conf. on Fusion Energy (San Diego, 2012), FTP/1-1, http://www-naweb.iaea.org/napc/physics/FEC/FEC2012/index.htm

[10] Terry J. et al., 2012 Proc. 24th Int. Conf. on Fusion Energy (San Diego, 2012), EX/P5-39, http://www-naweb.iaea.org/napc/physics/FEC/FEC2012/index.htm 
[11] Garrett M.L. et al., AIP Conference Proceedings 1406 (19th RF Topical Conference on RF Power in Plasmas, 2011) 203.

[12] Pütterich T. et al., Plasma Phys. Control. Fusion 50, 8 (2008) 085016.

[13] Dux R. et al., J. Nucl. Mater. 363-365 (2007) 112.

[14] Gunn J. et al, in Fusion Energy 2008 (Proc. 22nd Int. Conf. Geneva, 2008) (Vienna: IAEA), CD-ROM file EX/P6-32 and http://www-naweb.iaea.org/napc/physics/FEC/FEC2008/html/index.htm

[15] Colas L. et al., 2-Dimensional Mapping of ICRF-Induced Scrape-Off Layer Modifications with a Retarding Field Analyser on ASDEX Upgrade, submitted to 20th RF Topical Conference on RF Power in Plasmas, 2013.

[16] Kallenbach A. et al., IEEE Transactions on Plasma Science 40, 3 (2012), 605 -613

[17] Wukitch S. et al., in Fusion Energy 2010 (Proc. 23rd Int. Conf. Daejeon, 2010) (Vienna: IAEA), CD-ROM file EXD/P3-37 and http://www-naweb.iaea.org/napc/physics/FEC/FEC2010/index.htm

[18] Lancellotti V. et al., Nucl. Fusion 46 (2006) S476.

[19] Jaquet P. et al., Nucl. Fusion 52 (2012) 042002.

[20] Brambilla M., Plasma Phys. Control. Fusion 41 (1999) 1.

[21] Jaeger et. al, Phys. Plasmas 8 (2001) 1573.

[22] Eckstein W., Sputtering yields, in R. Behrisch, W. Eckstein (Eds.): Sputtering by Particle Bombardment, Topics Appl. Physics 110, 32186 (2007), Springer-Verlag Berlin Heidelberg 2007.

[23] Viezzer E. et al., Rev. Sci. Instrum. 83, (2012) 103501; doi: 10.1063/1.4755810

[24] Faugel H. and Bobkov V., Open source hard- and software: Using Arduino boards to keep old hardware running, Fusion Eng. Des., in press.

[25] Polozhiy K. et al., Europhysics Conference Abstracts 35G (2011), P4.071.

[26] Stober J. et al., 2012 Proc. 24th Int. Conf. on Fusion Energy (San Diego, 2012), EX/1-4, http://www-naweb.iaea.org/napc/physics/FEC/FEC2012/index.htm

[27] Schweinzer J. et al., 2012 Proc. 24th Int. Conf. on Fusion Energy (San Diego, 2012), EX/P2-03, http://www-naweb.iaea.org/napc/physics/FEC/FEC2012/index.htm

[28] Sips A.C.C. et al., 2012 Proc. 24th Int. Conf. on Fusion Energy (San Diego, 2012), ITR/P1-11, http://www-naweb.iaea.org/napc/physics/FEC/FEC2012/index.htm 